

\title{
AN ASYMPTOTIC-PRESERVING SCHEME FOR SYSTEMS OF CONSERVATION LAWS WITH SOURCE TERMS ON 2D UNSTRUCTURED MESHES
}

\author{
Christophe Berthon, Guy Moebs, \\ CÉline SARAZIN-Desbois and Rodolphe Turpault
}

\begin{abstract}
In this paper, finite volume numerical schemes are developed for hyperbolic systems of conservation laws with source terms. The systems under consideration degenerate into parabolic systems in large times when the source terms become stiff. In this framework, it is crucial that the numerical schemes are asymptoticpreserving, i.e., that they degenerate accordingly. Here, an asymptotic-preserving numerical scheme is proposed for any system within the aforementioned class on $2 \mathrm{D}$ unstructured meshes.

This scheme is proved to be consistent and stable under a suitable CFL condition. Moreover, we show that it is also possible to prove that it preserves the set of (physically) admissible states under a geometric property on the mesh. Finally, numerical examples are given to illustrate its behavior.
\end{abstract}

\section{Introduction}

The objective of this paper is to build a suitable numerical scheme for hyperbolic systems of conservation laws which can be written in the following form:

$$
\partial_{t} \boldsymbol{U}+\operatorname{div}(\boldsymbol{F}(\boldsymbol{U}))=\gamma(\boldsymbol{U})(\boldsymbol{R}(\boldsymbol{U})-\boldsymbol{U}), \quad(t, x) \in \mathbb{R}_{+} \times \mathbb{R}^{2} .
$$

Here, the Jacobian of the flux $\boldsymbol{F}$ is assumed to be diagonalizable in $\mathbb{R}$. The set of admissible states is denoted $\mathcal{A}$. Moreover, $\boldsymbol{R}$ is a smooth function of $\boldsymbol{U}$ such that for all $\boldsymbol{U} \in \mathcal{A}, \boldsymbol{R}(\boldsymbol{U}) \in \mathcal{A}$. Finally, $\gamma(\boldsymbol{U})$ is a positive real function which represents the stiffness of the source term.

The system (1) is assumed to fulfill the properties required in [6] so that it degenerates in long time and when the source term becomes stiff, more precisely when $\gamma(\boldsymbol{U}) t \rightarrow \infty$, into a parabolic system.

MSC2010: 35L65, 65M99, 65M08.

Keywords: Finite volume schemes, 2D unstructured mesh, asymptotic-preserving schemes, conservation laws with source terms, positivity-preserving schemes. 
There are numerous examples of such systems and two of them will be used throughout this article as illustrations, namely the isentropic Euler equations with friction and the $M_{1}$ model for radiative transfer:

Isentropic Euler with friction:

$$
\begin{array}{cc}
\boldsymbol{U}=\left(\begin{array}{c}
\rho \\
\rho u \\
\rho v
\end{array}\right), & \boldsymbol{F}(\boldsymbol{U})=\left(\begin{array}{cc}
\rho u & \rho v \\
\rho u^{2}+p(\rho) & \rho u v \\
\rho u v & \rho v^{2}+p(\rho)
\end{array}\right), \\
\gamma(\boldsymbol{U})=\kappa(\rho), & \boldsymbol{R}(\boldsymbol{U})=\left(\begin{array}{l}
\rho \\
0 \\
0
\end{array}\right),
\end{array}
$$

where $p$ is a smooth function such that $p^{\prime}(\rho)>0$. The set of admissible states is

$$
\mathcal{A}=\left\{\boldsymbol{U}=(\rho, \rho u, \rho v)^{\top} \in \mathbb{R}^{3} \mid \rho>0\right\} .
$$

The diffusion limit whenever $\kappa(\rho) t \rightarrow \infty$ is (see [8] for instance):

$$
\partial_{t} \rho-\operatorname{div}\left(\frac{1}{\kappa(\rho)} \nabla p(\rho)\right)=0 .
$$

$M_{1}$ model for radiative transfer (see [22] for the derivation of the model and [7] for the present form):

$$
\begin{array}{cc}
\boldsymbol{U}=\left(\begin{array}{c}
E \\
F x \\
F y \\
T
\end{array}\right), \quad \boldsymbol{F}(\boldsymbol{U})=\left(\begin{array}{cc}
F x & F y \\
c^{2} P x x & c^{2} P x y \\
c^{2} P y x & c^{2} P y y \\
0 & 0
\end{array}\right), \\
\gamma(\boldsymbol{U})=c \sigma_{m}(\boldsymbol{U}), \quad \boldsymbol{R}(\boldsymbol{U})=\left(\begin{array}{c}
\frac{\sigma(\boldsymbol{U}) a T^{4}+\sigma_{1}(\boldsymbol{U})}{\sigma_{m}(\boldsymbol{U})} \\
\frac{\sigma_{1}(\boldsymbol{U}) F x}{\sigma_{m}(\boldsymbol{U})} \\
\frac{\sigma_{1}(\boldsymbol{U}) F y}{\sigma_{m}(\boldsymbol{U})} \\
\frac{\sigma(\boldsymbol{U}) E+\sigma_{2}(\boldsymbol{U}) \rho C_{v} T}{\rho C_{v} \sigma_{m}(\boldsymbol{U})}
\end{array}\right),
\end{array}
$$

where

$$
\begin{aligned}
& P=E\left(\frac{1-\chi}{2} I_{d}+\frac{3 \chi-1}{2} \frac{F \otimes F}{\|F\|^{2}}\right), \\
& \chi=\chi\left(\xi=\frac{\|F\|}{c E}\right)=\frac{3+4 \xi^{2}}{5+2 \sqrt{4-3 \xi^{2}}}, \quad F=(F x, F y)^{\top},
\end{aligned}
$$


and

$$
\begin{aligned}
\sigma_{m}=\sigma_{m}(\boldsymbol{U}) & =\sigma(\boldsymbol{U}) \max \left(1, \frac{a T^{3}}{\rho C_{v}}\right), \\
\sigma_{1}(\boldsymbol{U}) & =\sigma_{m}(\boldsymbol{U})-\sigma(\boldsymbol{U}), \\
\sigma_{2}(\boldsymbol{U}) & =\sigma_{m}(\boldsymbol{U})-\sigma(\boldsymbol{U}) \frac{a T^{3}}{\rho C_{v}},
\end{aligned}
$$

The set of admissible states is

$$
\mathcal{A}=\left\{\boldsymbol{U}=\left(E, F_{x}, F_{y}, T\right)^{\top} \in \mathbb{R}^{4} \mid E>0, T>0,\|F\| \leq c E\right\} .
$$

When $\sigma_{m}(\boldsymbol{U}) t \rightarrow \infty$, the $M_{1}$ model degenerates into the so-called equilibrium diffusion equation:

$$
\partial_{t}\left(\rho C_{v} T+a T^{4}\right)-\operatorname{div}\left(\frac{c}{3 \sigma} \nabla a T^{4}\right)=0 .
$$

The main difficulty when designing a numerical scheme for such systems is to enforce the correct degeneracy in the diffusion limit. In other words, the limit of the scheme when $\gamma(\boldsymbol{U}) t \rightarrow \infty$ shall be a consistent approximation of the limit diffusion equation (see [28]). Obviously, this property is generally not fulfilled by numerical schemes hence the design of asymptotic-preserving (AP) schemes has been an important issue during the last decade.

For 1D applications, several asymptotic-preserving schemes were proposed in this context. The most explored way to do so is to use a modified HLL scheme [26] and cleverly control the numerical diffusion in the spirit of the work of Gosse and Toscani for the telegraph equations [25]. This technique has been widely used for the $M_{1}$ model for radiative transfer and Euler equations with friction (see for instance $[11 ; 12 ; 5 ; 14]$ ) and extended to general cases [7]. Other techniques have also been used, such as $[9 ; 15]$ in the framework of Euler equations with friction, or [3] where the knowledge of the convergence rates towards equilibrium is extensively used.

The situation is much more difficult for 2D applications however. While it is quite straightforward in the case of Cartesian grids (see [4] for example), the situation is far more complex on unstructured grids. One of the reasons is that the classical two-point flux scheme (or FV4 [24]) which is the target of many AP schemes is not consistent anymore. The only exception is the MPFA-based AP scheme for Friedrich systems developed in [13].

Our goal is therefore to propose an AP finite volume scheme for any system of the form (1). This scheme is a natural extension of the 1D scheme proposed in [7]. It will be proved to be consistent and stable under a natural unrestrictive CFL 
condition. Moreover, it is also possible to enforce the preservation of the set of admissible states provided a geometric property is satisfied by the mesh.

The rest of the paper is organized as follows. First the notation used throughout the paper is presented. Then, the scheme is introduced in the case where the mesh is admissible. This additional property indeed allows us to simplify several expressions. In this case, the scheme is proved to be consistent and to preserve the set of admissible states under a natural CFL condition. We explain how the AP property can be enforced.

Then, the scheme is extended for general meshes. Once again, it is proved to be consistent and AP. Since the target scheme in the diffusive regime is the diamond scheme [17] — which does not preserve the maximum principle — it does not preserve the set of admissible states on general meshes. However, we show that this feature can be recovered under some geometric constraint on the mesh.

Finally, the scheme is benchmarked on numerical examples and a few words on the optimization and parallelization of the code conclude this work.

\section{Notation}

Since we intend to provide a finite volume scheme which may be used in either cell-centered or vertex-centered (or cell-vertex) contexts, we will use the following nomenclature:

- The primary mesh $\mathcal{M}$ is the set of all control volumes (or cells) effectively used in the scheme. As a consequence, the primary mesh is the primal mesh in the context of cell-centered schemes and the dual mesh in the context of vertex-centered schemes.

- The secondary mesh is a set of control volumes defined around the nodes of the primary mesh. Practically, the secondary mesh is the dual mesh in the context of cell-centered schemes and the primal mesh in the context of vertex-centered schemes.

For the sake of clarity in the following, the primary mesh is simply called the mesh whenever no confusion is possible.

The notation used throughout this paper is summarized in Figure 1:

- $N_{K}$ is the number of nodes (and interfaces) of the cell $K \in \mathcal{M}$.

- $x_{K}$ is the centroid of the cell $K$.

- The nodes of the cell $K$ are locally denoted $\left\{A_{i}\right\}_{i=1 \ldots N_{K}}$ with the convention $A_{0}=A_{N_{K}}$ and $A_{N_{K}+1}=A_{1}$.

- The neighboring cells of the cell $K$ (i.e., cells that share an edge with $K$ ) are locally numbered from 1 to $N_{K}$ such that $K \cap L_{i}=\left[A_{i} A_{i+1}\right]$. Their centroids are locally denoted $\left\{x_{i}\right\}_{i=1 \ldots N_{K}}$. 

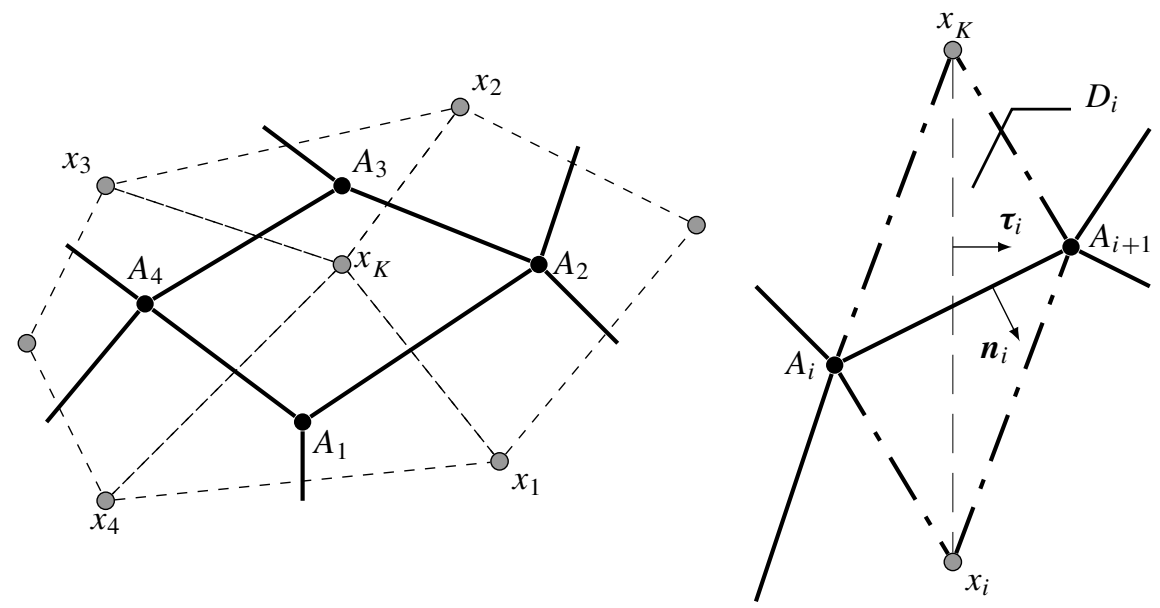

Figure 1. Local mesh notation. Left: one cell and the local notation. Right: the diamond cell associated with the $i$-th interface of the cell $K \in \mathcal{M}$.

- $d_{i}^{K}:=\left\|x_{K} x_{i}\right\|$.

- $e_{i}:=\left\|A_{i} A_{i+1}\right\|$ is the length of the $i$-th interface of the cell $K$.

- The unit outward normal to the $i$-th interface of the cell $K$ is denoted $\boldsymbol{n}_{i}$.

- The unit normal to $x_{K} x_{i}$ is denoted $\boldsymbol{\tau}_{i}$. As a convention, it is chosen such that $\left(\boldsymbol{n}_{i} \wedge \boldsymbol{\tau}_{i}\right) . O z>0$.

- The characteristic length $r^{K}$ is defined by $r^{K}:=|K| / p_{k}$ where $p_{k}=\sum_{i} e_{i}$ is the perimeter of $K$. Let us remark that for example, $r^{K}=h / 4$ in a square cell of size $h$.

For the sake of clarity, we recall that

$$
\boldsymbol{F} \cdot\left(\begin{array}{l}
n_{x} \\
n_{y}
\end{array}\right)=\left(\begin{array}{ll}
\boldsymbol{F}_{x} & \boldsymbol{F}_{y}
\end{array}\right) \cdot\left(\begin{array}{l}
n_{x} \\
n_{y}
\end{array}\right)=n_{x} \boldsymbol{F}_{x}+n_{y} \boldsymbol{F}_{y} .
$$

\section{An AP scheme on admissible meshes}

In this section, the scheme is introduced on admissible meshes (see definition below). This property indeed allows us to simplify the notation and to prove the preservation of admissible states. The extension to general meshes is described in the next section. Let us mention that this scheme is explicit but an implicit version can be obtained straightforwardly using the same technique.

Definition. The mesh is said to be admissible if

$$
x_{K} x_{i} \cdot A_{i} A_{i+1}=0 \quad \text { for all } K \text { and all } i \in\left[1, N_{K}\right],
$$

i.e., if all interfaces are orthogonal to the lines which join the centroids of the cells. 
Obviously, the property required for a mesh to be admissible restricts the generality of this class of meshes. However, it includes Cartesian grids and it is possible to consider unstructured admissible meshes for reasonable geometries. For instance, a mesh consisting of triangles in which the centroids are the circumcenters is often a valid possibility.

On admissible meshes, we propose to consider the following scheme:

$$
\begin{aligned}
\boldsymbol{U}_{K}^{n+1}=\boldsymbol{U}_{K}^{n}-\frac{\Delta t}{|K|} \sum_{i=1}^{N_{K}} e_{i} \alpha_{i}^{K} \mathcal{F}_{K, i}^{n} \cdot \boldsymbol{n}_{i} & +\frac{\Delta t}{|K|} \sum_{i=1}^{N_{K}} e_{i} \alpha_{i}^{K} \boldsymbol{F}\left(\boldsymbol{U}_{K}^{n}\right) \cdot \boldsymbol{n}_{i} \\
+ & \frac{\Delta t}{|K|} \sum_{i=1}^{N_{K}} e_{i} b_{i}^{K}\left(1-\alpha_{i}^{K}\right)\left(\boldsymbol{R}\left(\boldsymbol{U}_{K}^{n}\right)-\boldsymbol{U}_{K}^{n}\right),
\end{aligned}
$$

where $\alpha_{i}^{K}$ is defined by

$$
\alpha_{i}^{K}=\frac{b_{i}^{K}}{b_{i}^{K}+\gamma_{i}^{K} r^{K}},
$$

and the numerical flux is given by

$$
\mathcal{F}_{K, i}^{n}=\frac{1}{2}\left(\boldsymbol{F}\left(\boldsymbol{U}_{K}^{n}\right)+\boldsymbol{F}\left(\boldsymbol{U}_{i}^{n}\right)\right)-\frac{1}{2} b_{i}^{K}\left(\boldsymbol{U}_{i}^{n}-\boldsymbol{U}_{K}^{n}\right),
$$

where $b_{i}^{K}>0$ is a parameter larger that all characteristic speeds to be defined depending on the form of (1). This scheme is designed to keep the numerical diffusion in the normal direction to the interfaces in order to stay consistent in the diffusion limit. It is also designed to be a convex combination of $1 \mathrm{D}$ schemes that enter the formalism of [7]. As we will see in the proofs, this feature allows it to automatically inherit the preservation of admissible states under a suitable CFL condition.

Remark. For the $M_{1}$-model, since the physical flux is equal to zero in the last equation (therefore, the temperature $T$ is only coupled through the source term), the corresponding component of the numerical flux is also set to zero.

Theorem 1.1. Assume that $\gamma_{i}^{K}=\gamma>0$ is a constant. Then the scheme (8)-(10) is consistent with (1).

Proof. Let us consider a sequence of regular meshes such that

$$
\lim _{\eta_{K} \rightarrow 0} r^{K}=0
$$

where $\eta_{K}$ is the radius of the largest circle inside the cell $K$. This immediately implies that

$$
\lim _{\eta_{K} \rightarrow 0} \alpha_{i}^{K}=1
$$


Moreover, the divergence formula gives

$$
\sum_{i=1, N_{K}} e_{i} \boldsymbol{F}\left(\boldsymbol{U}_{K}^{n}\right) \cdot \boldsymbol{n}_{i}=0 .
$$

Therefore, the first two terms of (8) are consistent with the hyperbolic part of (1).

Now, the third term is consistent with the source term. Indeed,

$$
\left(1-\alpha_{k}^{K}\right)=\frac{\gamma r^{K}}{b_{i}^{K}+\gamma r^{K}}
$$

so

$$
\frac{1}{|K|} \sum_{i=1}^{N_{K}} e_{i} b_{i}^{K}\left(1-\alpha_{i}^{K}\right)\left(\boldsymbol{R}\left(\boldsymbol{U}_{K}^{n}\right)-\boldsymbol{U}_{K}^{n}\right)=\frac{1}{|K|} \sum_{i=1}^{N_{K}} e_{i} b_{i}^{K} \frac{\gamma r^{K}\left(\boldsymbol{R}\left(\boldsymbol{U}_{K}^{n}\right)-\boldsymbol{U}_{K}^{n}\right)}{b_{i}^{K}+\gamma r^{K}},
$$

which tends to $\gamma\left(\boldsymbol{R}\left(\boldsymbol{U}_{K}^{n}\right)-\boldsymbol{U}_{K}^{n}\right)$ when $\eta_{K} \rightarrow 0$.

Finally, the scheme (8)-(10) is consistent with (1).

Remark. This consistency result may be extended to include the case of nonconstant $\gamma$. Indeed, $\gamma$ only appears in the scheme inside $\alpha_{i}^{K}$. When $\gamma$ is not constant, one can perform a Taylor expansion of $\alpha_{i}^{K}$ and the above proof is still valid up to high order terms which tend to zero with $\eta_{K}$.

Theorem 1.2. The scheme preserves the set of admissible states as soon as the following CFL condition holds:

$$
\max _{K, i} b_{i}^{K} \frac{\Delta t}{|K|} p_{K} \leq 1 .
$$

Proof. To prove this result, we establish that the scheme (8)-(10) can be written as a convex combination of 1D schemes. These 1D schemes are nothing but the ones proposed in [7] with a Rusanov flux for the hyperbolic part.

Let $\boldsymbol{U}_{K, i}^{n+1}$ be an intermediate state given by such a $1 \mathrm{D}$ scheme in the normal direction to the interface $A_{i} A_{i+1}$ and considering a space length $r^{K}$. It is therefore given by

$$
\begin{array}{r}
\boldsymbol{U}_{K, i}^{n+1}=\boldsymbol{U}_{K, i}^{n}-\frac{\Delta t}{r^{K}}\left(\alpha_{i}^{K} \mathcal{F}_{K, i}^{n} \cdot \boldsymbol{n}_{i}-\alpha_{K}^{K} \mathcal{F}_{K, K}^{n} \cdot \boldsymbol{n}_{i}\right)-\frac{\Delta t}{r^{K}}\left(\alpha_{i}^{K}-\alpha_{K}^{K}\right) \boldsymbol{F}\left(\boldsymbol{U}_{K}^{n}\right) \cdot \boldsymbol{n}_{i} \\
+\frac{b_{i}^{K} \Delta t}{r^{K}}\left(\left(1-\alpha_{i}^{K}\right)+\left(1-\alpha_{K}^{K}\right)\right)\left(\boldsymbol{R}\left(\boldsymbol{U}_{K}^{n}\right)-\boldsymbol{U}_{K}^{n}\right),
\end{array}
$$

where $\alpha_{i}^{K}$ is given by (9) and $\alpha_{K}^{K}=1$.

According to [7], such a scheme preserves the convex set of admissible states $\mathcal{A}$ under the CFL condition

$$
\max _{K, i}\left(b_{i}^{K} \frac{\Delta t}{r^{K}}\right) \leq \frac{1}{2} .
$$


Now, we set

$$
\omega_{K, i}:=\frac{e_{i}}{p_{K}}
$$

so that $\omega_{K, i}$ is positive and

$$
\sum_{i=1}^{N_{K}} \omega_{K, i}=1 .
$$

Equipped with this choice, we consider the following convex combination of the 1D schemes (12):

$$
\begin{aligned}
\sum_{i=1}^{N_{K}} \omega_{K, i} \boldsymbol{U}_{K, i}^{n+1}=\sum_{i=1}^{N_{K}} \omega_{K, i} \boldsymbol{U}_{K, i}^{n}-\sum_{i=1}^{N_{K}} \omega_{K, i} \frac{\Delta t}{r^{K}}\left(\alpha_{i}^{K} \mathcal{F}_{K, i}^{n} \cdot \boldsymbol{n}_{i}-\alpha_{K}^{K} \mathcal{F}_{K, K}^{n} \cdot \boldsymbol{n}_{i}\right) \\
-\sum_{i=1}^{N_{K}} \omega_{K, i} \frac{\Delta t}{r^{K}}\left(\alpha_{i}^{K}-\alpha_{K}^{K}\right) \boldsymbol{F}\left(\boldsymbol{U}_{K}^{n}\right) \cdot \boldsymbol{n}_{i} \\
+\sum_{i=1}^{N_{K}} \omega_{K, i} \frac{b_{i}^{K} \Delta t}{r^{K}}\left(\left(1-\alpha_{i}^{K}\right)+\left(1-\alpha_{K}^{K}\right)\right)\left(\boldsymbol{R}\left(\boldsymbol{U}_{K}^{n}\right)-\boldsymbol{U}_{K}^{n}\right) .
\end{aligned}
$$

Since

$$
\frac{\omega_{K, i}}{r^{K}}=\frac{e_{i}}{|K|},
$$

this convex combination is nothing but the scheme (8), i.e.,

$$
\boldsymbol{U}_{K, i}^{n+1}=\sum_{i=1}^{N_{K}} \omega_{K, i} \boldsymbol{U}_{K, i}^{n+1},
$$

and the choice of $r^{K}$ implies that the CFL condition (13) becomes the condition (11). Therefore, if all $\boldsymbol{U}_{K}^{n} \in \mathcal{A}$, this condition ensures that all $\boldsymbol{U}_{K, i}^{n+1} \in \mathcal{A}$ and therefore $\boldsymbol{U}_{K}^{n+1} \in \mathcal{A}$ since $\mathcal{A}$ is convex.

At this point, the scheme still doesn't preserve the asymptotic. Hopefully, this property may be easily recovered in the same way it was enforced in [7]. Indeed, as mentioned in [7], for any $\bar{\gamma}$ such that $\gamma+\bar{\gamma}>0$ the scheme (8) may be applied to the system

$$
\partial_{t} \boldsymbol{U}+\operatorname{div}(\boldsymbol{F}(\boldsymbol{U}))=(\gamma+\bar{\gamma})(\overline{\boldsymbol{R}}(\boldsymbol{U})-\boldsymbol{U}),
$$

where

$$
\overline{\boldsymbol{R}}(\boldsymbol{U})=\frac{\gamma \boldsymbol{R}(\boldsymbol{U})+\bar{\gamma} \boldsymbol{U}}{\gamma+\bar{\gamma}}
$$


In fact, this system is equivalent to (1) but using the scheme (8) on it allows one to consider $\bar{\gamma}$ as a free parameter which can be used to recover the asymptotic limit.

The asymptotic limit of the scheme (8) is formally obtained by performing a Chapmann-Enskog expansion. A small parameter $\varepsilon$ is introduced and the following rescalings are made:

$$
\Delta t \leftarrow \frac{\Delta t}{\varepsilon}, \quad \gamma \leftarrow \frac{\gamma}{\varepsilon}
$$

Therefore we have

$$
\alpha_{i}^{K} \leftarrow \frac{\varepsilon b_{i}^{K}}{\varepsilon b_{i}^{K}+\left(\gamma_{i}^{K}+\overline{\gamma_{i}^{K}}\right) r^{K}}, \quad \text { and } \quad 1-\alpha_{i}^{K} \leftarrow \frac{\left(\gamma_{i}^{K}+\overline{\gamma_{i}^{K}}\right) r^{K}}{\varepsilon b_{i}^{K}+\left(\gamma_{i}^{K}+\overline{\gamma_{i}^{K}}\right) r^{K}} .
$$

Using these rescalings in (8), an identification gives:

terms in $\varepsilon^{-1}$ :

$$
\boldsymbol{R}\left(\boldsymbol{U}_{K}^{n}\right)=\boldsymbol{U}_{K}^{n},
$$

terms in $\varepsilon^{0}$ :

$$
\boldsymbol{U}_{K}^{n+1}=\boldsymbol{U}_{K}^{n}-\left.\frac{\Delta t}{|K|} \sum_{i=1}^{N_{K}} e_{i} \frac{b_{i}^{K}}{\left(\gamma_{i}^{K}+\overline{\gamma_{i}^{K}}\right) r^{K}}\left[\mathcal{F}\left(\boldsymbol{U}_{K}^{n}\right) \cdot \boldsymbol{n}_{i}-\boldsymbol{F}\left(\boldsymbol{U}_{K}^{n}\right) \cdot \boldsymbol{n}_{i}\right]\right|_{\mid \boldsymbol{R}\left(\boldsymbol{U}_{K}^{n}\right)=U_{K}^{n}} .
$$

The parameters $\overline{\gamma_{i}^{K}}$ are then fixed by imposing a given scheme for the diffusion limit. As examples, we propose two corrections that allow us to recover the classical two-point flux scheme in the asymptotic regimes of the $M_{1}$ model for radiative transfer and Euler system with friction.

Remark. Let us underline that the Chapmann-Enskog expansion is nothing but a tool to obtain the correct asymptotic limit in the diffusion regime. It has to be handled with care for other purposes since it assumes that both $\gamma$ and $\Delta t$ are large (with the same order in $\varepsilon$ ), while the degeneracy is indeed governed by $\gamma t$ (see for instance [8]).

However, techniques that avoid rescalings which can be used in the continuous case (for instance [8]) cannot be extended to the discrete level.

AP correction for the $M_{1}$ model. In the case of the $M_{1}$ model for radiative transfer (5) $b_{i}^{K}=c$ and the equilibrium (16) gives $F_{x}=F_{y}=0$ and $E=a T^{4}$. The sum of the first and fourth equations of (17) hence becomes

$$
\left(\rho C_{v}+a T^{4}\right)_{K}^{n+1}=\left(\rho C_{v} T+a T^{4}\right)_{K}^{n}+\frac{\Delta t}{|K|} \sum_{i=1}^{N_{K}} \frac{c e_{i}}{2\left(\sigma_{m, i}^{K}+\bar{\sigma}_{i}^{K}\right) r^{K}}\left(\left(a T^{4}\right)_{i}^{n}-\left(a T^{4}\right)_{K}^{n}\right) .
$$

This scheme is not consistent in general with the equilibrium diffusion equation (7), but it is possible to choose $\bar{\sigma}$ in order to recover the consistency in the diffusive 
limit. For example, if we take

$$
\left(\sigma_{m, i}^{K}+\bar{\sigma}_{i}^{K}\right)=\sigma_{m, i}^{K} \frac{3\left|D_{i}\right|}{2 r^{K} e_{i}}>0,
$$

then the limit scheme in the diffusion regime is

$$
\begin{aligned}
& \left(\rho C_{v} T+a T^{4}\right)_{K}^{n+1} \\
& \quad=\left(\rho C_{v} T+a T^{4}\right)_{K}^{n}+\frac{\Delta t}{|K|} \sum_{i=1}^{N_{K}} \frac{c e_{i}^{2}}{3 \sigma_{i}^{K}|D i|}\left(\left(a T^{4}\right)_{i}^{n}-\left(a T^{4}\right)_{K}^{n}\right),
\end{aligned}
$$

which is nothing but the classic FV4 scheme (see [24]) for the diffusion equation (7) on admissible meshes.

AP correction for Euler equations with friction. Now we consider the case of Euler equations with friction (2). In this case, the equilibrium (16) gives $\rho u=\rho v=0$, and the first equation of (17) hence becomes

$$
\rho_{K}^{n+1}=\rho_{K}^{n}+\frac{\Delta t}{|K|} \sum_{i=1}^{N_{K}} e_{i} \frac{\left(b_{i}^{K}\right)^{2}}{2\left(\kappa_{i}^{K}+\bar{\kappa}_{i}^{K}\right) r^{K}}\left(\rho_{i}^{n}-\rho_{K}^{n}\right) .
$$

Once again, this scheme is not consistent in general with the limit diffusive regime (4), however it is also possible to choose $\bar{\kappa}$ in order to recover the consistency in this limit. For instance if we take

$$
\left(\kappa_{i}^{K}+\bar{\kappa}_{i}^{K}\right)= \begin{cases}2 \kappa_{i}^{K} \frac{\left(b_{i}^{K}\right)^{2}\left|D_{i}\right|}{r^{K} e_{i}} \frac{\rho_{i}^{n}-\rho_{K}^{n}}{p\left(\rho_{i}^{n}\right)-p\left(\rho_{K}^{n}\right)} & \text { if } \rho_{i}^{n} \neq \rho_{K}^{n}, \\ 2 \kappa_{i}^{K} \frac{\left(b_{i}^{K}\right)^{2}\left|D_{i}\right|}{r^{K} e_{i} p^{\prime}\left(\rho_{K}^{n}\right)} & \text { otherwise, }\end{cases}
$$

then the limit scheme in the diffusion regime is

$$
\rho_{K}^{n+1}=\rho_{K}^{n}+\frac{\Delta t}{|K|} \sum_{i=1}^{N_{K}} \frac{e_{i}^{2}}{\kappa_{i}^{K}\left|D_{i}\right|}\left(p\left(\rho_{i}^{n}\right)-p\left(\rho_{K}^{n}\right)\right),
$$

which is consistent with the diffusion equation (4) on admissible meshes since it is once again nothing but the FV4 scheme in this context.

Remark. It is required for the hyperbolicity of the system that $p$ be an increasing function of $\rho$. Therefore, the choice (20) provides positive values for $\left(\kappa_{i}^{K}+\bar{\kappa}_{i}^{K}\right)$, as required in robustness theorems.

\section{Extension to more general meshes}

When the mesh is not admissible, there is an additional difficulty since the classical two-point finite volume scheme (also known as FV4 [24]) is not consistent with the 
diffusion equation anymore. The target scheme in the diffusive limit must therefore properly take into account the whole gradient. For the sake of consistency and simplicity, we choose to use the same gradient discretization in the hyperbolic part. Among the possible choices available in the literature, we adopt the diamond scheme strategy [17], but other strategies could be considered such as DDFV schemes [27; $18]$ (see also $[1 ; 2 ; 10 ; 16]$ ) or hybrid strategies (see $[19 ; 20 ; 23]$ and references therein).

With the diamond scheme to approximate the gradients, it is possible to propose a natural extension of the scheme for admissible meshes (8)-(10) into the following generalized scheme:

$$
\begin{aligned}
\boldsymbol{U}_{K}^{n+1}=\boldsymbol{U}_{i}^{n}+\frac{\Delta t}{|K|} \sum_{i=1}^{N_{K}} e_{i} \alpha_{K, i}^{n} \mathcal{F}_{K, i}^{n} & \cdot \boldsymbol{n}_{i}+\frac{\Delta t}{|K|} \sum_{i=1}^{N_{K}} e_{i} \alpha_{K, i}^{n} \boldsymbol{F}\left(\boldsymbol{U}_{K}^{n}\right) \cdot \boldsymbol{n}_{i} \\
+ & \frac{\Delta t}{|K|} \sum_{i=1}^{N_{K}} e_{i}\left(1-\alpha_{K, i}^{n}\right) b_{i}^{K}\left(\boldsymbol{R}\left(\boldsymbol{U}_{K}^{n}\right)-\boldsymbol{U}_{K}^{n}\right),
\end{aligned}
$$

where

$$
\mathcal{F}_{K, i}^{n}=\frac{1}{2}\left(\boldsymbol{F}\left(\boldsymbol{U}_{K}^{n}\right)+\boldsymbol{F}\left(\boldsymbol{U}_{i}^{n}\right)\right)-\frac{1}{2} b_{i}^{K} \theta_{i}^{K} \nabla_{i}^{K} \boldsymbol{U}_{K}^{n} \cdot \boldsymbol{n}_{i}
$$

with $\theta_{i}^{K}$ a positive parameter to be specified later and

$$
\nabla_{i}^{K} \boldsymbol{U}_{K}^{n} \cdot \boldsymbol{n}_{i}=\frac{\boldsymbol{U}_{i}^{n}-\boldsymbol{U}_{K}^{n}}{2\left|D_{i}\right|} e_{i}+\frac{\boldsymbol{U}_{A_{i+1}}^{n}-\boldsymbol{U}_{A_{i}}^{n}}{2\left|D_{i}\right|} d_{i}^{K} \boldsymbol{n}_{i} \cdot \boldsymbol{\tau}_{i},
$$

$\boldsymbol{U}_{A_{i}}^{n}$ being the value of the solution at the node $A_{i}$ (see Figure 1). This value is obtained as a mean value of the solution in the cells which share $A_{i}$ as a node (see [17]).

With this definition, we immediately see that the scheme for admissible meshes (8)-(10) is recovered if $\theta_{i}^{K}=2\left|D_{i}\right| / e_{i}$.

Theorem 2.1. Assume that $\gamma_{i}^{K}=\gamma>0$ is a constant and $\theta_{i}^{K} \rightarrow 0$ when $\eta_{K} \rightarrow 0$. Then the scheme (22)-(23) is consistent with (1).

Proof. As was pointed in the introduction of the scheme (22)-(23), the only difference compared to the scheme designed for admissible meshes (8)-(10) lies in the definition of the discrete gradient in the numerical flux. But if $\theta_{i}^{K} \rightarrow 0$, this difference between the two schemes converges to zero when $\eta_{K} \rightarrow 0$. Therefore, the arguments in the proof of Theorem 1.1 can still be applied to obtain the consistency.

The preservation of the set of admissible states $\mathcal{A}$ is all the more difficult since most finite volume schemes for parabolic problems do not preserve the maximum principle. Only a few examples ensure this property [29;21]. It is therefore expected that the extension of Theorem 1.2 either does not hold for nonadmissible meshes 
or is very difficult to prove. Interestingly, it is sometimes possible to recover the maximum principle under some geometric condition on the mesh.

Definition. The mesh is said to be $\delta$-admissible if there exists a constant $\delta>0$ such that the following property holds:

$$
\forall K \in \mathcal{M}, \forall i \in\left[1, N_{K}\right], \quad 1+\frac{e_{i-1} \overline{d_{i-1}^{K}}}{e_{i}^{2}} \frac{\left|D_{i}\right|}{3\left|D_{i}-1\right|}-\frac{e_{i+1} \overline{d_{i+1}^{K}}}{e_{i}^{2}} \frac{\left|D_{i}\right|}{3\left|D_{i}+1\right|}>\delta,
$$

where

$$
\overline{d_{i}^{K}}=d_{i}^{K} \boldsymbol{n}_{i} \cdot \boldsymbol{\tau}_{i}
$$

Remark. With this definition, an admissible mesh is $\delta$-admissible for all $\delta \leq 1$ since all $d_{i}^{K}$ are then equal to 0 . While not all meshes are $\delta$-admissible, this condition turned out to be satisfied by most of the meshes we tested that were generated with reasonable constraints on the angles.

Equipped with this definition, we can obtain a generalization of Theorem 1.2 for the scheme (22)-(23) applied on a $\delta$-admissible mesh where the secondary mesh is made of triangles (e.g., vertex-centered schemes of a triangular mesh).

Theorem 2.2. Assume that the mesh is $\delta$-admissible and that the secondary mesh is made of triangles. Let us also assume that $\alpha_{i}^{K}$ is constant inside each cell $K \in \mathcal{M}$ $\left(\alpha_{i}^{K}=\alpha^{K}\right)$ and set $\theta_{i}^{K}=2\left|D_{i}\right| / \delta e_{i}$.

Then, the scheme (22)-(23) preserves the set of admissible states $\mathcal{A}$ as long as the following CFL condition holds:

$$
\max _{K \in \mathcal{A}, i \leq N_{K}}\left\{b_{i}^{K} \theta_{i}^{K} \delta_{i}^{K}\right\} \frac{\Delta t}{|K|} p_{K} \leq \frac{1}{2} .
$$

Proof. We consider here secondary meshes made of triangles. This characteristic allows us to give a simple expression from the extrapolated solution at the vertices of the primary mesh (see Figure 1):

$$
\boldsymbol{U}_{A_{i}}=\frac{1}{3}\left(\boldsymbol{U}_{K}+\boldsymbol{U}_{i}+\boldsymbol{U}_{i-1}\right)
$$

Then, from the expression of the numerical flux (23), we obtain

$$
\begin{aligned}
\sum_{i=1}^{N_{K}} e_{i} \mathcal{F}_{K, i}^{n} \cdot \boldsymbol{n}_{i}= & \frac{1}{2} \sum_{i=1}^{N_{K}} e_{i}\left(\boldsymbol{F}\left(\boldsymbol{U}_{K}^{n}\right)+\boldsymbol{F}\left(\boldsymbol{U}_{i}^{n}\right)\right) \cdot \boldsymbol{n}_{i} \\
& -\frac{1}{2} \sum_{i=1}^{N_{K}} e_{i} b_{i}^{K} \theta_{i}^{K}\left(\frac{\boldsymbol{U}_{i}^{n}-\boldsymbol{U}_{K}^{n}}{2\left|D_{i}\right|} e_{i}+\frac{\boldsymbol{U}_{i+1}^{n}-\boldsymbol{U}_{K}^{n}+\boldsymbol{U}_{K}^{n}-\boldsymbol{U}_{i-1}^{n}}{6\left|D_{i}\right|} \overline{d_{i}^{K}}\right) .
\end{aligned}
$$


Then, summing by parts to reorganize the terms $\boldsymbol{U}_{i \pm 1}^{n}-\boldsymbol{U}_{K}^{n}$ into $\boldsymbol{U}_{i}^{n}-\boldsymbol{U}_{K}^{n}$ we get

$$
\sum_{i=1}^{N_{K}} e_{i} \mathcal{F}_{K, i}^{n} \cdot \boldsymbol{n}_{i}=\frac{1}{2} \sum_{i=1}^{N_{K}} e_{i}\left(\boldsymbol{F}\left(\boldsymbol{U}_{K}^{n}\right)+\boldsymbol{F}\left(\boldsymbol{U}_{i}^{n}\right)\right) \cdot \boldsymbol{n}_{i}-\frac{1}{2} \sum_{i=1}^{N_{K}} e_{i} b_{i}^{K} \theta_{i}^{K} \delta_{i}^{K}\left(\boldsymbol{U}_{i}^{n}-\boldsymbol{U}_{K}^{n}\right),
$$

where

$$
\delta_{i}^{k}=\frac{e_{i}}{2\left|D_{i}\right|}+\frac{e_{i-1}}{e_{i}} \frac{\overline{d_{i-1}^{K}}}{6\left|D_{i}-1\right|}-\frac{e_{i+1}}{e_{i}} \frac{\overline{d_{i+1}^{K}}}{6\left|D_{i}+1\right|} .
$$

With the choice $\theta_{i}^{K}=2\left|D_{i}\right| / \delta e_{i}$ and since the mesh is $\delta$-admissible, we have $\theta_{i}^{K} \delta_{i}^{K} \geq 1$ for all $K \in \mathcal{M}$ and all $i \in\left[1, N_{K}\right]$. Moreover, the numerical flux can be expressed as

$$
\mathcal{F}_{K, i}^{n} \cdot \boldsymbol{n}_{i}=\frac{1}{2}\left(\boldsymbol{F}\left(\boldsymbol{U}_{K}^{n}\right)+\boldsymbol{F}\left(\boldsymbol{U}_{i}^{n}\right)\right) \cdot \boldsymbol{n}_{i}-\frac{1}{2} b_{i}^{K} \theta^{K} \delta_{i}^{K}\left(\boldsymbol{U}_{i}^{n}-\boldsymbol{U}_{K}^{n}\right) .
$$

Hence the scheme (22)-(23) can be recast as a convex combination of 1D schemes as in the proof of Theorem 1.2. These 1D schemes are Rusanov schemes with a speed of $b_{i}^{K} \theta_{i}^{K} d_{i}^{K} \geq b_{i}^{K}$ from which the CFL condition follows:

$$
\max _{K \in \mathcal{A}, i \leq N_{K}}\left\{b_{i}^{K} \theta_{i}^{K} \delta_{i}^{K}\right\} \frac{\Delta t}{|K|} p_{K} \leq \frac{1}{2} .
$$

Remark. We have several comments concerning this theorem:

- The choice of $\theta_{i}^{K}$ tends to 0 when $\eta_{K} \rightarrow 0$ as it was requested for the sake of consistency.

- A similar theorem may be obtained on more general meshes. However, the geometric condition (equivalent to the definition of $\delta$-admissible meshes above) quickly becomes cumbersome.

- The main restriction is to consider $\alpha_{i}^{K}$ that are constant per cell. As one can guess from the $\alpha_{i}^{K}$ chosen to obtain AP schemes in the previous section, it is not always possible to select a correction $\overline{\gamma_{i}^{K}}$ such that $\gamma_{i}^{K}+\overline{\gamma_{i}^{K}}>0$ and does not depend on $i$.

- Other choices of $\theta_{i}^{K}$ allow one to recover the same result. For instance, one can consider $\theta_{i}^{K}=\max _{i \leq N_{K}} 2\left|D_{i}\right| / 2 \delta$.

The scheme (22)-(23) is also not asymptotic preserving in general but the procedure previously used can still be considered in order to recover this property. Indeed, a formal Chapmann-Enskog expansion will lead to the same two relations (16) and (17). Of course, in the last relation, the numerical flux is given by (23). Now, the correction is once again illustrated in the example of the $M_{1}$ model for radiative transfer and Euler equations with friction. The objective is to recover an extension of the schemes obtained in the diffusive limit in the case of admissible meshes. 
AP correction for the $M_{1}$ model. We first consider the $M_{1}$ model for radiative transfer (5). We recall that in this case $b_{i}^{K}=c$ and the equilibrium (16) gives $F_{x}=F_{y}=0$ and $E=a T^{4}$. The sum of the first and fourth equations of (17)-(23) hence becomes

$$
\left(\rho C_{v}+a T^{4}\right)_{K}^{n+1}=\left(\rho C_{v} T+a T^{4}\right)_{K}^{n}+\frac{\Delta t}{|K|} \sum_{i=1}^{N_{K}} \frac{c^{2} e_{i}}{2\left(\sigma_{m, i}^{K}+\bar{\sigma}_{i}^{K}\right) r^{K}} \nabla_{i}^{K}\left(a T^{4}\right)^{n} .
$$

Once again, this scheme is not consistent in general with the equilibrium diffusion equation (7) but it is possible to choose $\bar{\sigma}$ in order to recover the consistency in the diffusive limit. For example, if we take

$$
\left(\sigma_{m, i}^{K}+\bar{\sigma}_{i}^{K}\right)=\sigma_{m, i}^{K} \frac{3 c \theta_{i}^{K}}{2 r^{K}}>0,
$$

then the limit scheme in the diffusion regime is

$$
\left(\rho C_{v} T+a T^{4}\right)_{K}^{n+1}=\left(\rho C_{v} T+a T^{4}\right)_{K}^{n}+\frac{\Delta t}{|K|} \sum_{i=1}^{N_{K}} \frac{c e_{i}}{3 \sigma_{i}^{K}} \nabla_{i}^{K}\left(a T^{4}\right)_{i}^{n},
$$

which is consistent with the diffusion equation (7) and a clear extension of (19). Moreover, if $\sigma$ is a constant and $\theta_{i}^{K}=\theta^{K}$ then $\bar{\sigma}_{i}^{K}=\bar{\sigma}^{K}$ and Theorem 2.2 can be applied. In order to meet such a requirement, one may choose the form of $\theta_{i}^{K}$ already mentioned earlier: $\theta_{i}^{K}=\max _{i \leq N_{K}} \frac{2\left|D_{i}\right|}{2 \delta}$.

AP correction for Euler equations with friction. If we consider Euler equations with friction (2), the equilibrium (16) gives $\rho u=\rho v=0$ and the first equation of (17)-(23) hence becomes

$$
\rho_{K}^{n+1}=\rho_{K}^{n}+\frac{\Delta t}{|K|} \sum_{i=1}^{N_{K}} e_{i} \frac{\left(b_{i}^{K}\right)^{2} \theta_{i}^{K}}{2\left(\kappa_{i}^{K}+\bar{\kappa}_{i}^{K}\right) r^{K}} \nabla_{i}^{K} \rho^{n} \cdot \boldsymbol{n}_{i} .
$$

As previously, this scheme is not consistent in general with the limit diffusive regime (4), however it is possible to choose $\bar{\kappa}$ in order to recover the consistency in this limit. For instance if we take

$$
\left(\kappa_{i}^{K}+\bar{\kappa}_{i}^{K}\right)=2 \kappa_{i}^{K} \frac{\left(b_{i}^{K}\right)^{2} \theta_{i}^{K}}{r^{K}} \frac{\nabla_{i}^{K} \rho^{n} \cdot \boldsymbol{n}_{i}}{\nabla_{i}^{K} p\left(\rho^{n}\right) \cdot \boldsymbol{n}_{i}},
$$

then the limit scheme in the diffusion regime is

$$
\rho_{K}^{n+1}=\rho_{K}^{n}+\frac{\Delta t}{|K|} \sum_{i=1}^{N_{K}} \frac{e_{i}}{\kappa_{i}^{K}} \nabla_{i}^{K} p\left(\rho^{n}\right) \cdot \boldsymbol{n}_{i},
$$

which is consistent with the diffusion equation (4) and a direct extension of (21). 
Remark. The choice (27) also provides positive values for $\left(\kappa_{i}^{K}+\bar{\kappa}_{i}^{K}\right)$ since $p$ is required to be an increasing function of $\rho$ for the sake of hyperbolicity.

However, this choice generates an $\alpha_{i}^{K}$ which is not constant per cell and therefore, Theorem 2.2 cannot be applied here.

\section{Numerical results}

Validation tests. Validation tests are performed in this subsection in order to illustrate the behavior of the scheme. All tests share this same setup: a Riemann problem for the $M_{1}$ model for radiative transfer is considered on the rectangular domain $[0,5] \times[0,1]$, with

$$
\left(E, F_{x}, F_{y}, T\right)^{\top}(0, x)= \begin{cases}\left(a T_{L}^{4}, c f_{x, L} a T_{L}^{4}, 0, T_{L}\right)^{\top} & \text { if } x<1, \\ \left(a T_{R}^{4}, 0,0, T_{R}\right)^{\top} & \text { otherwise. }\end{cases}
$$

In the following, $T_{L}=10000, T_{R}=300$ and $f_{x, L}=0$ unless otherwise specified and $\rho C_{v}=10^{-2}$. We also recall that $c=3 \times 10^{8}$.

All tests are performed at least with $\sigma=0$ and $\sigma=1$. Indeed, when $\sigma=0$, the model turns out to be a hyperbolic system and the preservation of admissible states is expected to be more difficult than in the presence of the (regularizing) source term.

The end times of the simulations are $t=2 \times 10^{-9}$ when $\sigma=0$ and $t=1 \times 10^{-8}$ when $\sigma=1$.

The approximate solutions are computed on two different meshes: a coarse one (5152 triangles) and a fine one (132 006 triangles). Both are $\delta$-admissible and their optimal values of $\delta$ are $\delta_{1}=1.095$ for the coarse grid and $\delta_{2}=5.599 \times 10^{-2}$ for the fine one.

In practice, these meshes would be called "very coarse" and "coarse", since there are only respectively 35 and 160 cells in the $x$ direction. These choices are made in order to easily visualize the errors made by the schemes on the graphs.

Finally, the solutions are compared to reference solutions. When $\sigma=0$, the reference solution is the exact solution of the corresponding 1D Riemann problem (see [30]). When $\sigma \neq 0$, the exact solution is not available so the reference solution is given by the grid-converged 1D asymptotic-preserving scheme described in [7].

Figure 2 shows the computed solutions along $x=\frac{1}{2}$ compared with the exact solution in the case $\sigma=0$. Here, the conservation of admissible states is enforced by using $\theta_{i}^{K}=\max _{i \leq N_{K}} 2\left|D_{i}\right| / 2 \delta$ where $\delta=\delta_{1}$ on the coarse mesh and $\delta_{2}$ on the fine one. The solution computed on the coarse grid is comparable to a 1D Rusanov scheme with a similar number of cells. On the other hand, since $\delta_{2} \ll \delta_{1}$, the numerical diffusion of the scheme is much larger on the fine mesh than on the coarse one. As a consequence, the approximation is better on the coarse grid in this case. 

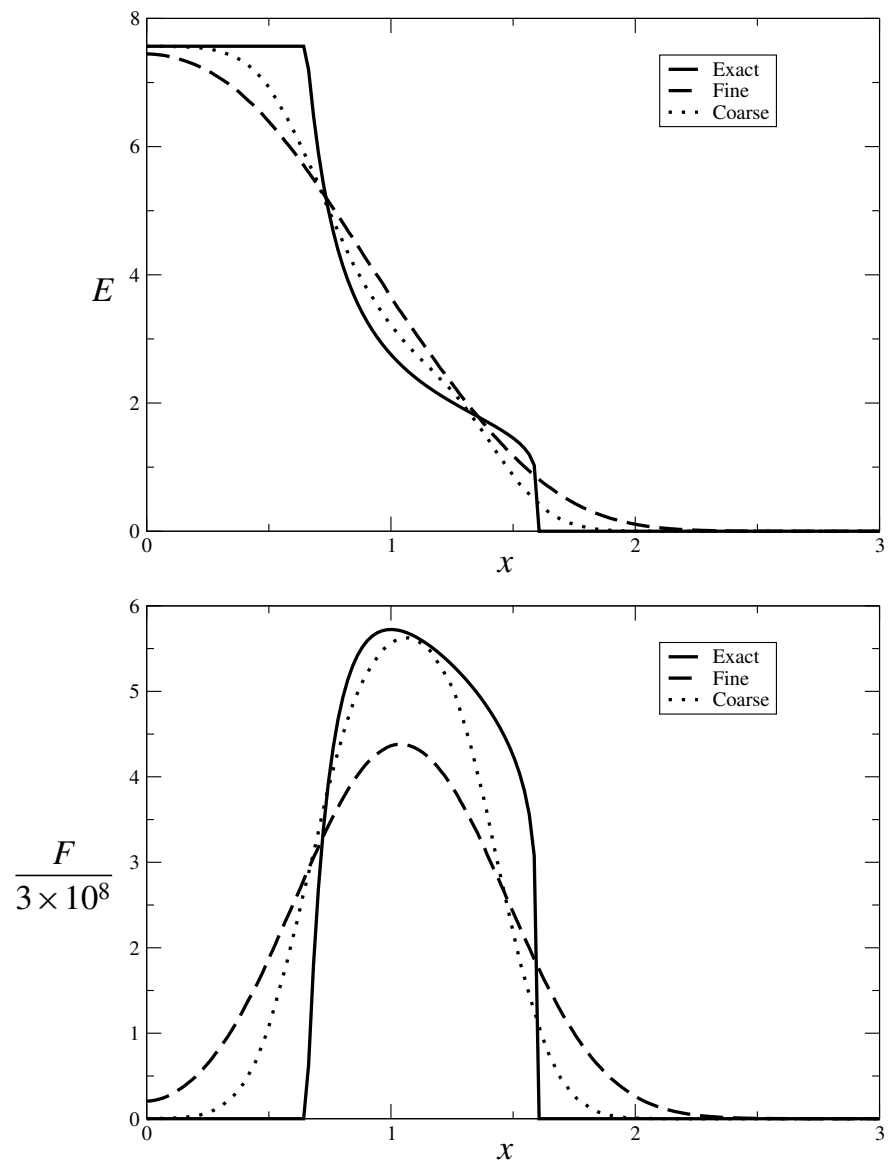

Figure 2. Exact and computed solutions along $x=\frac{1}{2}$ with $\sigma=0$. Top: $E$. Bottom: $F$.

Now, if we set $\delta=\delta_{1}$ on the fine mesh, the quality of the approximation behaves as expected, i.e., the approximation is better on the fine grid (see Figure 3). Here, even if the condition required to preserve the set of admissible states is violated, the setup is not stiff enough to beget unphysical values in the scheme.

The same conclusions can be drawn when $\sigma=1$ (see Figure 4), though the overall quality of the scheme is better than when $\sigma=0$. Indeed, the scheme is designed to recover the diamond scheme in the limit, which is a better approximation of the equilibrium diffusion equation than the Rusanov scheme for the hyperbolic part. This is particularly true when $\theta_{i}^{K}$ is large. Therefore, the quality of the approximation is expected to increase with $\sigma$.

Finally, the asymptotic-preserving property is clarified through a last validation example. This time, we fix $\sigma=1000$ and $t=2 \times 10^{-9}$ and the results shown in Figure 5 are compared with a grid-converged 1D approximation of the equilibrium diffusion 


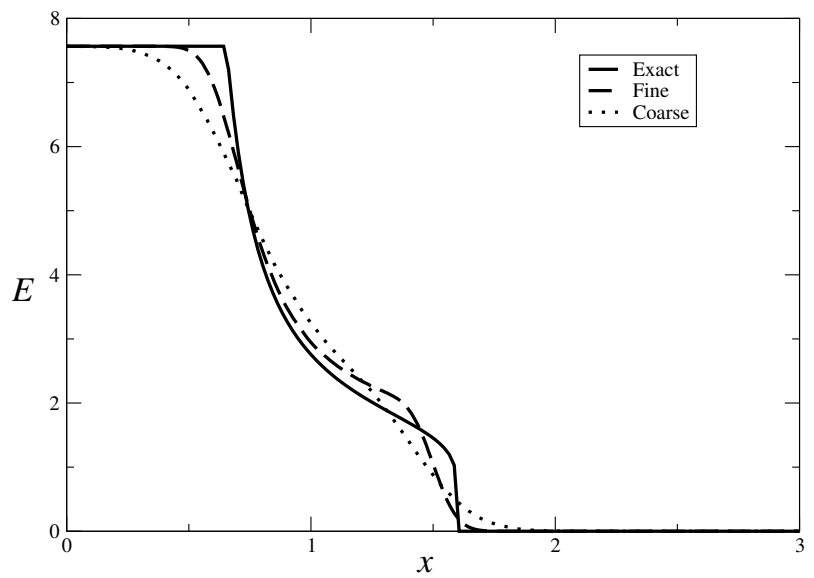

Figure 3. Reference and computed solutions along $x=\frac{1}{2}$ with $\sigma=0$ and $\delta=\delta_{1}$.
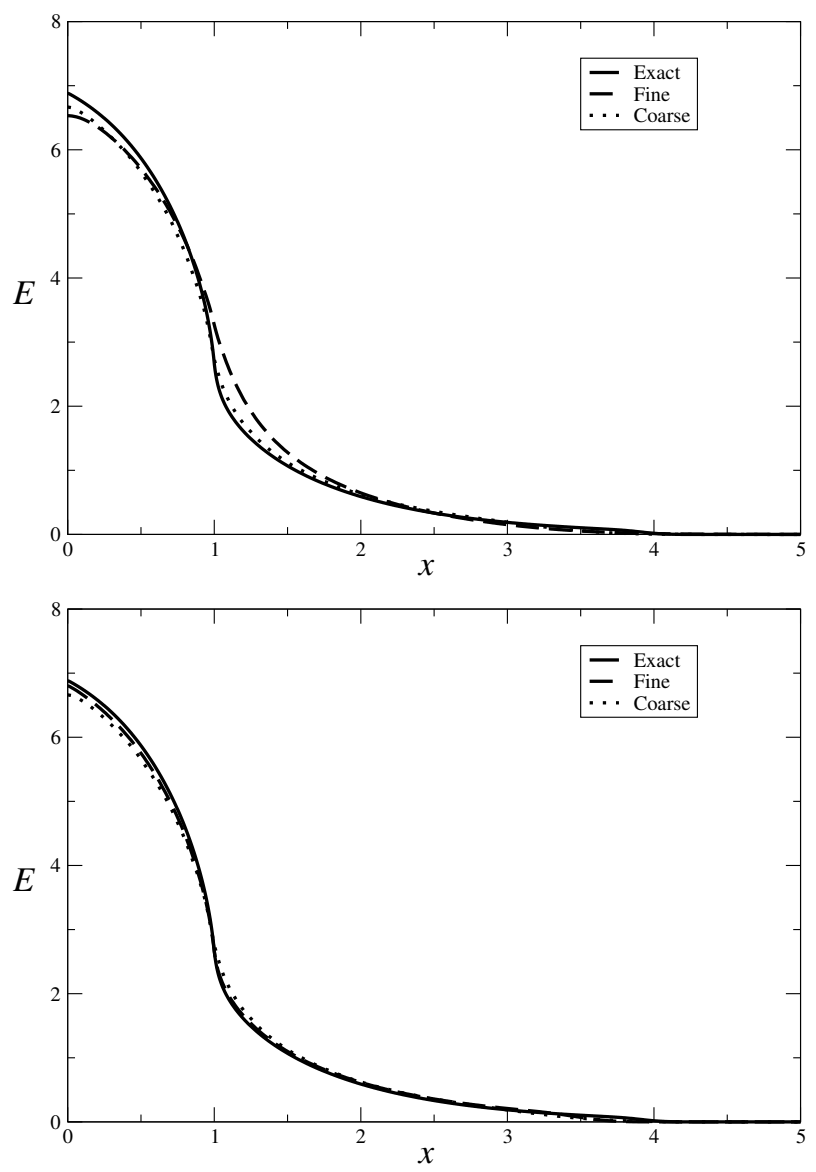

Figure 4. Reference and computed solutions along $x=\frac{1}{2}$ with $\sigma=1$. Top: optimal choice of $\delta$ to enforce the preservation of $\mathcal{A}$. Bottom: same choice of $\delta$ for both schemes. 

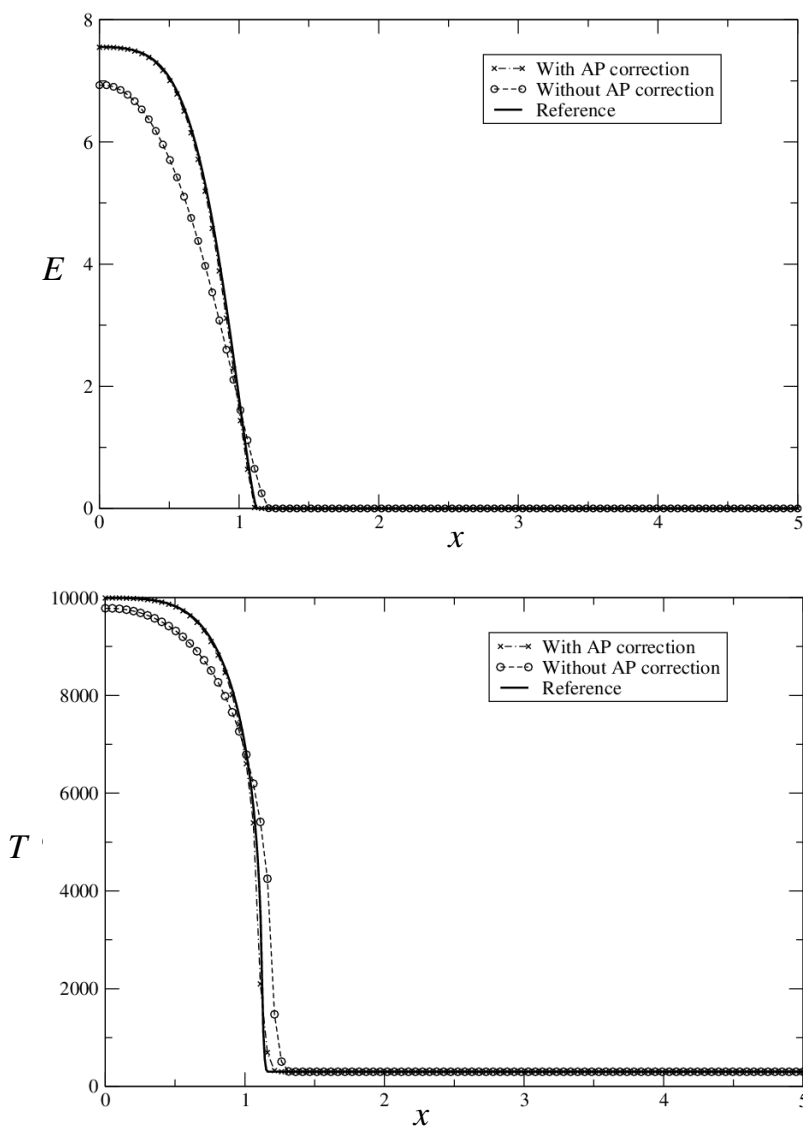

Figure 5. Reference and computed solutions with and without AP correction along $x=\frac{1}{2}$ with $\sigma=1000$. Top: $E$. Bottom: $T$.

equation. The tests are performed with and without the asymptotic-preserving correction on the fine grid to see the impact of the asymptotic preservation. We immediately see that with the AP correction, the scheme provides an approximation which is nearly indistinguishable from the reference solution. On the other hand, as expected, if the AP correction is turned off (i.e., $\overline{\gamma^{K}}=0$ ), there is a large discrepancy between the computed and reference solutions.

Radiative flow in a channel. In this subsection, a test case involving the evolution of radiation in a channel with multiple obstacles is performed. The setup is the following (see Figure 6). The entry condition on the left side of the channel models a beam of high energy $\left(F_{L}=c E_{L}=c a \times 10^{16}\right)$ compared to the initial state of the domain $\left(F_{0}=0, E_{0}=a \times 10^{4}\right)$. The opacity $\sigma$ is set to 1 and the $M_{1}$ model for radiative transfer is used. Moreover, 11 obstacles (with wall boundary conditions) 


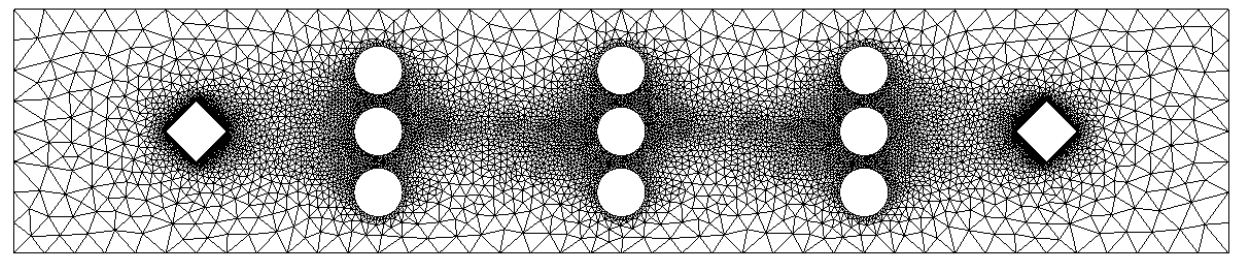

Figure 6. Mesh used for the radiative flow in a channel.

are scattered in the channel. A vertex-centered approached was used on a mesh consisting of 15348 cells refined near the obstacles.

Results for the energy $E$ and Eddington's factor $\chi$ at time $t=10^{-7}$ are shown on Figure 7. Let us emphasize that this case is numerically very challenging and that it is all the more critical to preserve the set of admissible states here since very small numerical errors may yield negative values for $E$ or values of $F>c E$, which immediately cause the code to crash. Indeed, several values of $\theta_{i}^{K}$ were tested in order to investigate the optimality of the conditions in Theorem 2.2 and even a value $5 \%$ larger than the choice stated in the theorem produces inadmissible results. In this sense, it seems that the condition of Theorem 2.2 is optimal.

Optimization and parallelization. Let us conclude this work with a few words on the implementation of the scheme. First, in order to reduce the distance between a given element of the mesh and its neighbors, a renumbering procedure should be considered. We used a classic Reverse Cuthill-McKee procedure as preprocessing. Since it greatly reduces the L2 data cache misses, a significant gain may be observed. As an example, on the mesh used in the previous simulation, the code ran for $293 \mathrm{~s}$ without the renumbering and $235 \mathrm{~s}$ when renumbering was activated. This time included the Cuthill-McKee algorithm, so the gain is even better if another run is made on the same mesh.

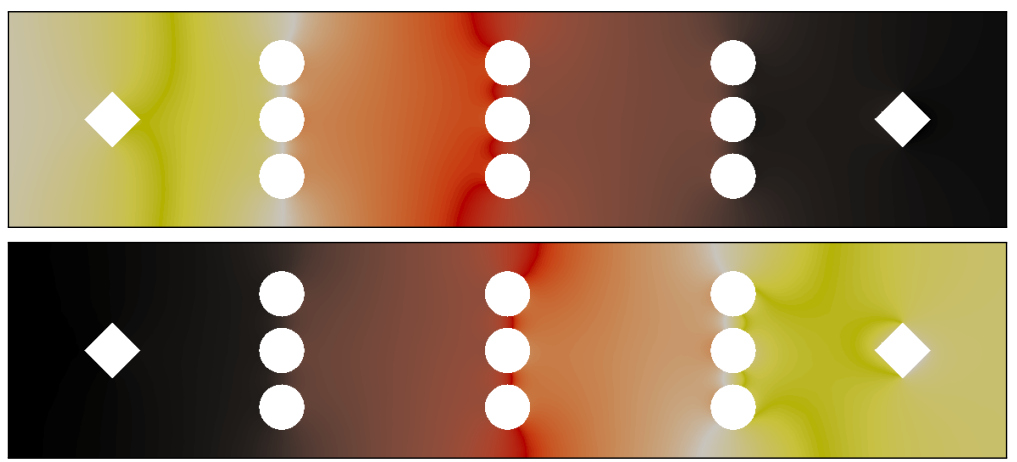

Figure 7. Radiative flow in a channel. Top: $E$. Bottom: $\chi$. 


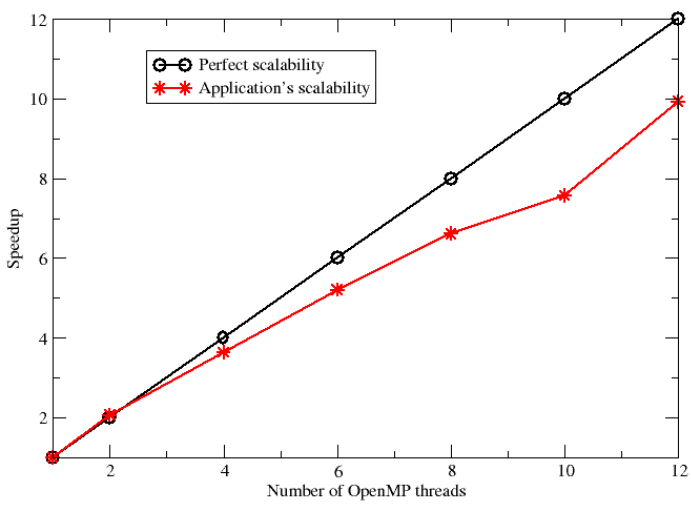

Figure 8. Scalability of the code.

Furthermore, since the scheme is explicit, the updates of the unknowns are independent from one another. Therefore, it is quite straightforward to parallelize the algorithm using OpenMP instructions (see http://openmp.org). On the one hand, such a strategy isn't suitable for massively parallel computations, but on the other hand it is very efficient on shared memory units and hence well adapted to multicore processors which are nowadays very common, even on personal computers. All the computations described above were run on a hexacore biprocessor node and a speed-up of 10 was obtained using all 12 cores of the machine. Figure 8 shows the scalability of the code on an example.

Finally, as it is classical for finite volume schemes, a loop on the edges is used (rather than on the elements for instance). In order to optimize the balance of work asked of each thread, we used an edge-based domain decomposition. This choice may save $20 \%$ or more time compared with an element domain decomposition since the number of edges per element is not constant. Table 1 shows the impact of such a choice on a representative example. Here, thread 6 has roughly $25 \%$ more work to do than thread 11 .

\begin{tabular}{|c|c|c|c|c|c|c|c|c|c|}
\hline 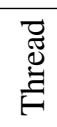 & \multicolumn{2}{|c|}{ elmt-based dec. } & \multicolumn{2}{|c|}{ edge-based dec. } & 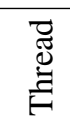 & \multicolumn{2}{|c|}{ elmt-based dec. } & \multicolumn{2}{|c|}{ edge-based dec. } \\
\hline 0 & 5550 & 33879 & 5492 & 33303 & 6 & 5550 & 37032 & 4967 & 33301 \\
\hline 1 & 5550 & 34713 & 5293 & 33303 & 7 & 5550 & 35200 & 5197 & 33304 \\
\hline 2 & 5551 & 31906 & 5806 & 33301 & 8 & 5551 & 33475 & 5461 & 33300 \\
\hline 3 & 5550 & 34391 & 5378 & 33300 & 9 & 5550 & 31999 & 5715 & 33305 \\
\hline 4 & 5550 & 32865 & 5588 & 33304 & 10 & 5550 & 30538 & 6004 & 33299 \\
\hline 5 & 5551 & 34282 & 5448 & 33299 & 11 & 5551 & 29338 & 6285 & 33299 \\
\hline
\end{tabular}

Table 1. Impact of the domain decomposition strategy: element-based decomposition versus edge-based decomposition. 


\section{References}

[1] B. Andreianov, M. Bendahmane, and K. H. Karlsen, Discrete duality finite volume schemes for doubly nonlinear degenerate hyperbolic-parabolic equations, J. Hyperbolic Differ. Equ. 7 (2010), no. 1, 1-67. MR 2011d:65241 Zbl 1207.35020

[2] B. Andreianov, F. Boyer, and F. Hubert, Discrete duality finite volume schemes for Leray-Lionstype elliptic problems on general 2D meshes, Numer. Methods Partial Differential Equations 23 (2007), no. 1, 145-195. MR 2008c:65283 Zbl 1111.65101

[3] D. Aregba-Driollet, M. Briani, and R. Natalini, Time asymptotic high order schemes for dissipative BGK hyperbolic systems, Numerische Mathematik (2015), 1-33 (English).

[4] C. Berthon, J. Dubois, B. Dubroca, T.-H. Nguyen-Bui, and R. Turpault, A free streaming contact preserving scheme for the $M_{1}$ model, Adv. Appl. Math. Mech. 2 (2010), no. 3, 259-285. MR 2011g:85009 Zbl 1262.65097

[5] C. Berthon, P. Charrier, and B. Dubroca, An HLLC scheme to solve the $M_{1}$ model of radiative transfer in two space dimensions, J. Sci. Comput. 31 (2007), no. 3, 347-389. MR 2008f:85004 Zbl 1133.85003

[6] C. Berthon, P. G. LeFloch, and R. Turpault, Late-time/stiff-relaxation asymptotic-preserving approximations of hyperbolic equations, Math. Comp. 82 (2013), no. 282, 831-860. MR 3008840 Zbl 1317.65182

[7] C. Berthon and R. Turpault, Asymptotic preserving HLL schemes, Numer. Methods Partial Differential Equations 27 (2011), no. 6, 1396-1422. MR 2838300 Zbl 1237.65100

[8] S. Bianchini, B. Hanouzet, and R. Natalini, Asymptotic behavior of smooth solutions for partially dissipative hyperbolic systems with a convex entropy, Comm. Pure Appl. Math. 60 (2007), no. 11, 1559-1622. MR 2010i:35227 Zbl 1152.35009

[9] F. Bouchut, H. Ounaissa, and B. Perthame, Upwinding of the source term at interfaces for Euler equations with high friction, Comput. Math. Appl. 53 (2007), no. 3-4, 361-375. MR 2008e:76120 Zbl 1213.76123

[10] F. Boyer and F. Hubert, Finite volume method for $2 D$ linear and nonlinear elliptic problems with discontinuities, SIAM J. Numer. Anal. 46 (2008), no. 6, 3032-3070. MR 2009f:65231 Zbl 1180.35533

[11] C. Buet and S. Cordier, An asymptotic preserving scheme for hydrodynamics radiative transfer models: numerics for radiative transfer, Numer. Math. 108 (2007), no. 2, 199-221. MR 2008i: 76138 Zbl 1127.76032

[12] C. Buet and B. Despres, Asymptotic preserving and positive schemes for radiation hydrodynamics, J. Comput. Phys. 215 (2006), no. 2, 717-740. MR 2007j:85005 Zbl 1090.76046

[13] C. Buet, B. Després, and E. Franck, Design of asymptotic preserving finite volume schemes for the hyperbolic heat equation on unstructured meshes, Numer. Math. 122 (2012), no. 2, 227-278. MR 2969268 Zbl 1263.65085

[14] C. Chalons, F. Coquel, E. Godlewski, P.-A. Raviart, and N. Seguin, Godunov-type schemes for hyperbolic systems with parameter-dependent source: the case of Euler system with friction, Math. Models Methods Appl. Sci. 20 (2010), no. 11, 2109-2166. MR 2011m:65179 Zbl 1213.35034

[15] C. Chalons, M. Girardin, and S. Kokh, Large time step and asymptotic preserving numerical schemes for the gas dynamics equations with source terms, SIAM J. Sci. Comput. 35 (2013), no. 6, A2874-A2902. MR 3138111 Zbl 1284.35262 
[16] Y. Coudière and G. Manzini, The discrete duality finite volume method for convection-diffusion problems, SIAM J. Numer. Anal. 47 (2010), no. 6, 4163-4192. MR 2011e:65231 Zbl 1210. 65183

[17] Y. Coudière, J.-P. Vila, and P. Villedieu, Convergence rate of a finite volume scheme for a two-dimensional convection-diffusion problem, M2AN Math. Model. Numer. Anal. 33 (1999), no. 3, 493-516. MR 2000f:65086 Zbl 0937.65116

[18] K. Domelevo and P. Omnes, A finite volume method for the Laplace equation on almost arbitrary two-dimensional grids, M2AN Math. Model. Numer. Anal. 39 (2005), no. 6, 1203-1249. MR 2006j:65312 Zbl 1086.65108

[19] J. Droniou, R. Eymard, T. Gallouët, and R. Herbin, A unified approach to mimetic finite difference, hybrid finite volume and mixed finite volume methods, Math. Models Methods Appl. Sci. 20 (2010), no. 2, 265-295. MR 2011d:65318 Zbl 1191.65142

[20] J. Droniou, R. Eymard, T. Gallouet, and R. Herbin, Gradient schemes: a generic framework for the discretisation of linear, nonlinear and nonlocal elliptic and parabolic equations, Math. Models Methods Appl. Sci. 23 (2013), no. 13, 2395-2432. MR 3109434 Zbl 1281.65136

[21] J. Droniou and C. Le Potier, Construction and convergence study of schemes preserving the elliptic local maximum principle, SIAM J. Numer. Anal. 49 (2011), no. 2, 459-490. MR 2012e:65237 Zbl 1227.65100

[22] B. Dubroca and J.-L. Feugeas, Étude théorique et numérique d'une hiérarchie de modèles aux moments pour le transfert radiatif, C. R. Acad. Sci. Paris Sér. I Math. 329 (1999), no. 10, 915-920. MR 2000h:85005 Zbl 0940.65157

[23] R. Eymard, T. Gallouët, and R. Herbin, Discretization of heterogeneous and anisotropic diffusion problems on general nonconforming meshes SUSHI: a scheme using stabilization and hybrid interfaces, IMA J. Numer. Anal. 30 (2010), no. 4, 1009-1043. MR 2012d:65246 Zbl 1202.65144

[24] R. Eymard, T. Gallouët, and R. Herbin, Finite volume methods, Handbook of numerical analysis, 7: Solutions of equations in $\mathbb{R}$ (part 3) and Techniques of scientific computing (part 3) (P. G. Ciarlet and J. L. Lions, eds.), vol. VII, North-Holland, Amsterdam, 2000, pp. 713-1020. MR 2002e:65138 Zbl 0981.65095

[25] L. Gosse and G. Toscani, An asymptotic-preserving well-balanced scheme for the hyperbolic heat equations, C. R. Math. Acad. Sci. Paris 334 (2002), no. 4, 337-342. MR 2003b:65087 Zbl 0996.65093

[26] A. Harten, P. D. Lax, and B. van Leer, On upstream differencing and Godunov-type schemes for hyperbolic conservation laws, SIAM Rev. 25 (1983), no. 1, 35-61. MR 85h:65188 Zbl 0565.65051

[27] F. Hermeline, Approximation of diffusion operators with discontinuous tensor coefficients on distorted meshes, Comput. Methods Appl. Mech. Engrg. 192 (2003), no. 16-18, 1939-1959. MR 2004d:65121 Zbl 1037.65118

[28] S. Jin and C. D. Levermore, Numerical schemes for hyperbolic conservation laws with stiff relaxation terms, J. Comput. Phys. 126 (1996), no. 2, 449-467. MR 97g:65173 Zbl 0860.65089

[29] C. Le Potier, Correction non linéaire et principe du maximum pour la discrétisation d'opérateurs de diffusion avec des schémas volumes finis centrés sur les mailles, C. R. Math. Acad. Sci. Paris 348 (2010), no. 11-12, 691-695. MR 2012a:65294 Zbl 1193.65188

[30] C. Sarazin-Desbois, Méthodes numériques pour des systèmes hyperboliques avec terme source provenant de physiques complexes autour du rayonnement, Thèse de Doctorat, Université de Nantes Angers Le Mans, 2013. 
Received January 17, 2014. Revised March 17, 2015.

CHRISTOPHE BERTHON: christophe.berthon@univ-nantes.fr

Université de Nantes, Laboratoire de Mathématiques Jean Leray, 2 rue de la Houssinière, 44322 Nantes Cedex 3, France

GUY MoEBS: guy.moebs@univ-nantes.fr

Université de Nantes, Laboratoire de Mathématiques Jean Leray, 2 rue de la Houssinière, 44322 Nantes Cedex 3, France

CÉlINE SARAZIN-DESBOIS: celine.sarazin@univ-nantes.fr

Université de Nantes, Laboratoire de Mathématiques Jean Leray, 2 rue de la Houssinière, 44322 Nantes Cedex 3, France

RODOLPHE TURPAULT: rodolphe.turpault@u-bordeaux.fr

Bordeaux-INP, Institut de Mathématiques de Bordeaux, 351 cours de la Libération, 33400 Talence, France 


\title{
Communications in Applied Mathematics and Computational Science
}

\author{
msp.org/camcos
}

EDITORS

MANAGING EDITOR

John B. Bell

Lawrence Berkeley National Laboratory, USA

jbbell@lbl.gov

\section{BOARD OF EDITORS}

\begin{tabular}{|c|c|c|c|}
\hline Marsha Berger & $\begin{array}{l}\text { New York University } \\
\text { berger@cs.nyu.edu }\end{array}$ & Ahmed Ghoniem & $\begin{array}{l}\text { Massachusetts Inst. of Technology, USA } \\
\text { ghoniem@mit.edu }\end{array}$ \\
\hline Alexandre Chorin & $\begin{array}{l}\text { University of California, Berkeley, USA } \\
\text { chorin@math.berkeley.edu }\end{array}$ & Raz Kupferman & $\begin{array}{l}\text { The Hebrew University, Israel } \\
\text { raz@math.huji.ac.il }\end{array}$ \\
\hline Phil Colella & $\begin{array}{l}\text { Lawrence Berkeley Nat. Lab., USA } \\
\text { pcolella@lbl.gov }\end{array}$ & Randall J. LeVeque & $\begin{array}{l}\text { University of Washington, USA } \\
\text { rj1@ amath.washington.edu }\end{array}$ \\
\hline Peter Constantin & $\begin{array}{l}\text { University of Chicago, USA } \\
\text { const@cs.uchicago.edu }\end{array}$ & Mitchell Luskin & $\begin{array}{l}\text { University of Minnesota, USA } \\
\text { luskin@umn.edu }\end{array}$ \\
\hline Maksymilian Dryja & $\begin{array}{l}\text { Warsaw University, Poland } \\
\text { maksymilian.dryja@acn.waw.pl }\end{array}$ & Yvon Maday & $\begin{array}{l}\text { Université Pierre et Marie Curie, France } \\
\text { maday@ann.jussieu.fr }\end{array}$ \\
\hline M. Gregory Forest & $\begin{array}{l}\text { University of North Carolina, USA } \\
\text { forest@amath.unc.edu }\end{array}$ & James Sethian & $\begin{array}{l}\text { University of California, Berkeley, USA } \\
\text { sethian@ math.berkeley.edu }\end{array}$ \\
\hline Leslie Greengard & $\begin{array}{l}\text { New York University, USA } \\
\text { greengard@ cims.nyu.edu }\end{array}$ & Juan Luis Vázquez & $\begin{array}{l}\text { Universidad Autónoma de Madrid, Spain } \\
\text { juanluis.vazquez@uam.es }\end{array}$ \\
\hline Rupert Klein & $\begin{array}{l}\text { Freie Universität Berlin, Germany } \\
\text { rupert.klein@pik-potsdam.de }\end{array}$ & Alfio Quarteroni & $\begin{array}{l}\text { Ecole Polytech. Féd. Lausanne, Switzerland } \\
\text { alfio.quarteroni@epfl.ch }\end{array}$ \\
\hline \multirow[t]{2}{*}{ Nigel Goldenfeld } & $\begin{array}{l}\text { University of Illinois, USA } \\
\text { nigel@uiuc.edu }\end{array}$ & Eitan Tadmor & $\begin{array}{l}\text { University of Maryland, USA } \\
\text { etadmor@cscamm.umd.edu }\end{array}$ \\
\hline & & Denis Talay & $\begin{array}{l}\text { INRIA, France } \\
\text { denis.talay@inria.fr }\end{array}$ \\
\hline
\end{tabular}

\section{PRODUCTION}

production@msp.org

Silvio Levy, Scientific Editor

See inside back cover or msp.org/camcos for submission instructions.

The subscription price for 2016 is US $\$ 95 /$ year for the electronic version, and $\$ 135 /$ year $(+\$ 15$, if shipping outside the US) for print and electronic. Subscriptions, requests for back issues from the last three years and changes of subscribers address should be sent to MSP.

Communications in Applied Mathematics and Computational Science (ISSN 2157-5452 electronic, 1559-3940 printed) at Mathematical Sciences Publishers, 798 Evans Hall \#3840, c/o University of California, Berkeley, CA 94720-3840, is published continuously online. Periodical rate postage paid at Berkeley, CA 94704, and additional mailing offices.

CAMCoS peer review and production are managed by EditFLOW ${ }^{\circledR}$ from MSP.

\section{PUBLISHED BY}

mathematical sciences publishers

nonprofit scientific publishing

http://msp.org/

(C) 2016 Mathematical Sciences Publishers 


\section{Communications in Applied Mathematics and Computational Science}

A front-tracking shock-capturing method for two gases

Mehdi Vahab and Gregory H. Miller

Identifying turbulent structures through topological segmentation

Peer-Timo Bremer, Andrea Gruber, Janine C. Bennett,

Attila Gyulassy, Hemanth Kolla, Jacqueline H. Chen and

RAY W. GROUT

An asymptotic-preserving scheme for systems of conservation laws with source terms on 2D unstructured meshes

Christophe Berthon, Guy Moebs, Céline Sarazin-Desbois and Rodolphe Turpault

An immersed boundary method for rigid bodies

Bakytzhan Kallemov, Amneet Pal Singh Bhalla, Boyce E.

Griffith and Aleksandar DoneV 